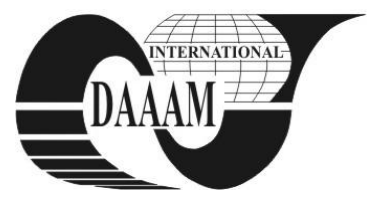

Annals of DAAAM for 2011 \& Proceedings of the 22nd International DAAAM Symposium, Volume 22, No. 1, ISSN 1726-9679 ISBN 978-3-901509-83-4, Editor B. Katalinic, Published by DAAAM International, Vienna, Austria, EU, 2011 Make Harmony between Technology and Nature, and Your Mind will Fly Free as a Bird Annals \& Proceedings of DAAAM International 2011

\title{
TRENDS IN THE FIELD OF HIGH PERFORMANCE COMPUTING AND THEIR INFLUENCE ON HPC FIELD
}

\author{
NOVACESCU, F[lorica]
}

\begin{abstract}
HPC (High Performance Computing) has become essential for the acceleration of innovation and the companies' assistance in creating new inventions, better models and more reliable products as well as obtaining processes and services at low costs. Solving complex problems of industry requires not only the most advanced hardware, but also some sophisticated software. Consequently, in this paper, will be presented the tendencies in the high performance computing field and their influence on the HPC field.
\end{abstract}

Key words: HPC, parallel computing, scientific computing, trends

\section{INTRODUCTION}

The increasing requirements of computing resources have determined two directions of development. On the one hand, the massive parallel supercomputers which have represented and they still represent the actual frontier in the field of numerical simulation of different physical phenomena. On the other hand, the so-called clusters which represent the standard of hardware endowment for universities and research institutes, although they are composed of performant units at the level of personal computers or work stations, they represent an extremely important potential of computing resources and which is hardly used in the present.

The development and maturation of numeric methods and their implementation algorithms constitutes an undoubted achievement of the last two decades. Simoultaneously, the available hardware resources have substantially increased the speed of calculation and the memory capacity, facilitating the current use of the numerical simulation of flows, analysis and optimization. The numeric experiment proved to be cheaper and faster than the physical experiment and the quality and the corresponding confidence rating of the numeric results has succeeded to convince more and more users. No plane, no car, no high-speed train, no turbine enters production without a preliminary CFD analysis.

After all, research and development are used in order to achieve scientific progress, for which HPC is only an instrument which is sometimes blunt, always expensive, but, however, it is an important instrument.

We live in a period interested in the use of the high performance calculation and a period with promises of unequalled performance for those who can effectively use the high performance computing.

This paper reports the latest developments and trends in the field of high performance scientific computing. Discussions of new theory and novel applications of HPC have been organized into four parts.

The information in this paper focuses particularly on: description the field of high performance scientific computing, parallel computing, scientific computing, parallel computers, and trends in the hpc field, presented here reveal important new directions toward the realization of a high performance computational society.

\section{THE FIELD OF HIGH PERFORMANCE COMPUTING}

The work field could be translated in computing in science and engineering (CSE) or computational science and engineering (CiSE).

From the soft point of view, the high performance computing field (HPC) means the parallel computing (computer science) and the scientific computing (applied Mathematics)

Regarding the hard branch we discover more branches of the computer science engineering which belong to architecture and communications. If we discuss cluster or computing centre and not the supercomputer as a finite product, the more we have to discuss the problems of operation systems, parallel filesystems, large data basis (VLDB multi-index, multiresolution), job scheduler, profiling, storage solutions (network filesystems, etc.). There is a lot of work in this field, and the problems are far from being solved.

\section{TENDENCIES IN HPC}

It seems that generally the HPC field is still in a continuous change, having a definite and stressed orientation on distributed computing with parallelism and other technologies for the optimization of performance aspects. Summarizing, there are the following results: the term "parallel" is not sufficient any more and all the aspects must be dedicated to performance, both memorization and communications are as important as computing, and to make the system easier to use, more flexible, thus, the performance should be obtained.

Taking into account that the progress will depend, in most cases on the possibility to achieve larger (or faster) simulations, it results that the computer simulation is essential for the scientific discovery, the approach of the society's challenges but also for the competitiveness of industry, thus, resulting that the general tendency in HPC is represented by the multiscale and multiphysical simulations, this can be achieved with the help of the larger supercomputers (which have a higher computing power, larger memory, a better storage, visualisation, etc), the simulation codes and instruments having the possibility to benefit of such systems, but also with the help of specialists in HPC, numeric analysis and in the practical phenomenon of their simulation working together.

The major challenges implied by the HPC are: performance, the total property cost (the cost of electric energy), programming, scalability and reliability. In the case of Petaflop systems, the use of accelerators is recommended, which are faster than processors because they give the users complete control on the programming of more units of parallel data and on the data transfer between memories and caches (D.B. Kirk \& W.W. Hwu, 2010). Some specific languages for accelerators being CUDA (Compute Unified Device Architecture) for graphic accelerators from Nvidia, Clearspeed SDK, etc. (Liu et al., 2009). 
Consequently, it results that the use of the graphic plates as matrix coprocessor is a trend now and it will become a standard. The use of CUDA technology is a reference point in this sense.

The graphic processing units (GPU) offer extraordinary performances in many applications of high performance computing. CUDA is both an architecture of parallel computing developed by NVIDIA, and a computing motor in graphics processing units NVIDIA, whose interface is available for programmers of programming languages such as $\mathrm{C}$ (Jason Sanders \& Edward Kandrot, 2010). The CUDA technology can be used in order to solve mathematical problems in a short time, which could not be easily solved before, problems which can be involved in fields such as: image and video processing, biology and computational Chemistry, fluids dynamics simulations, imge reconstruction, seismic analysis, ray tracing, and many more. GPUs offer different computing compromises in comparison to the traditional multicore systems, and also offer a top bandwidth for access to a larger memory (W.W. Hwu, 2010).

When we generally discuss the supercomputers, the first aspect is that refering to their increased processing capacity and another aspect is that refering to a large number of high performance processors. Supercomputers generally offer high performances with the frequent need of maintenance. They consume a large quantity of energy, thus, generating a large warmth quantity. This overheating of processors sometimes leads to a higher rate of system failure. Consequently, the processors must be echeloned and immense cooling systems must be installed in the room where the supercomputer is placed. Today, when the world goes towards "green", the concept of green supercomputing is widely spread and initiates a real balance between the performance and reliability of the supercomputer. Consequently, we can state that the energetic efficiency constitutes a major aspect of the HPC field, there is a certain trend of "green computing" having in view the reduction of energetic resources (supercomputing using as little energetic resources as possible). In order to achieve the improvement of the execution and energetic efficiency, we need to progress in diverse fields, such as: program optimization, the optimization of the program planning and the reduction of the energetic power during the empty running of the system components for all the levels of the system hierarchy.

It is recommended that every time when we speak about the supercomputers in list Top500 to refer to their position in the list Green500, too, which could help the research community in the field HPC and also different businessmen to focus their attention on other factors (such as the energy consumption), not only on the performance which concerns the computing speed (the computing speed remains one of the most important factors which must be taken into account in the HPC field).

Another trend in HPC is that of supercomputing in small spaces, namely, the reduction of the physical dimension of supercomputers.

Concerning the processors and accelerators, some future tendencies result refering to the extension of the number of cores per chip, namely that they aim to achieve chips with a larger and larger number of cores of small dimensions and specific hardware capable of graphic operations. Consequently, the stress is placed on the hybrid architectures which combine optimized processors for sequential processing with optimized accelerators for parallel processing. This combination exploits the fact that the applications have usually both parts, namely, the sequential part which can be effectively executed by fast sequential processors and the parallel part which is effectively executed by massive-parallel accelerators. Generally, as compared to the homogenous multicore systems, the hybrid architectures offer better balance between performance and used resources. Hybrid platforms have won and still win continuous popularity on a large scale due to the competitive price of GPU as compared to accelerators and due to their capacity to provide a higher computing rate and a larger memory bandwidth. Consequently, using such platforms we can obtain significant speedups as compared to the actual homogenous multicore systems in the same price range.

The increase of the optic interconnections number also represents a trend. It is intended to integrate photonic cells on chip and the use of optic interconnection.

The increasing importance of data and IO devices leads to the orientation on the computing centre type data-centric, distributed and parallel files systems, stress on data integrity.

Heterogenous configuration is another important aspect used for example in the case of clusters.

\section{CONCLUSIONS}

The field of high performance scientific computing is at the crossroads of a number of disciplines and sets of scientific abilities (in the sense that it requires at least elementary knowledge and competences in the respective scientific fields). Computing comes from an applicative context, consequently, knowledge from Physics and engineering sciences will be required, thus, the denomination ,computing in science and engineering" appeared. Then, the problems of these applicative fields are to be transformed in problems of liniar algebra and sometimes in theory of combinations, in order to be subject to implementation, it is obvious that knowledge of numeric analysis, liniar algebra and discrete mathematics is required.

In order to complete the aspects refering to the implications of this field, besides the ones already mentioned, it is required to have an understanding of the computer's architecture, both at CPU level and parallel computing level (Ananth Grama et al., 2003).

Taking into account all the aspects mentioned above, aspects involved in the HPC field, a major problem is that they did not reach a combination of all these aspects in a single one, in the sense that while there is documentation in the specialised literature refering to applicative Physics, parallel computing but also the performance optimization, no book succeeded to group these components in a unified and harmonious way which could clearly define and describe the field of high performance scientific computing.

Basically, this work represents a synthesis of the latest trends identified in the HPC field. This helps focus on the important aspects of HPC field, and efficient use of HPC tool help create an efficient computational world for many areas such as those already mentioned above. Also, the important aspects of HPC help create new research directions opposite the HPC such as sustainable energy research, geothermal energy, natural hazards, new energies, and so on.

\section{REFERENCES}

Ananth Grama, Anshul Gupta, George Karypis, Vipin Kumar (2003). Introduction to Parallel Computing, 2nd edition, Addison-Wesley, ISBN 0-201-64865-2

D.B. Kirk and W.W. Hwu (2010). Programming Massively Parallel Processors: A Hands-on Approac, Morgan Kaufmann, ISBN-13: 978-0123814722

Jason Sanders, Edward Kandrot (2010). CUDA by Example. An Introduction to general - purpose GPU Programming, Addison-Wesley Professional, ISBN-13: 978-0-13-1387683

Liu, D. Maskell, and B. Schmidt (2009). CUDASW++: Optimizing Smith-Waterman Sequence Database Searches for CUDA-enabled Graphics Processing Units, BMC Research Notes, 2(1):73

W.W. Hwu (2010). GPU Computing Gems, Morgan Kaufmann, ISBN: 9780123849885 